\title{
REVIEW
}

\section{A review of published reports on neuroprotection in spinal cord injury}

\author{
G Onose ${ }^{1}$, A Anghelescu ${ }^{1}$, DF Muresanu ${ }^{2}$, L Padure ${ }^{3}$, MA Haras ${ }^{1}$, CO Chendreanu ${ }^{1}$, LV Onose ${ }^{4}$, A Mirea ${ }^{1}$, \\ AV Ciurea ${ }^{5}$, WS El Masri ${ }^{6}$ and KRH von Wild ${ }^{7}$ \\ ${ }^{1}$ The Clinic Division of Physical \& Rehabilitation Medicine, Emergency Hospital 'Bagdasar-Arseni', Bucharest, Romania; ${ }^{2}$ The \\ Neurological Clinic of the 'Iuliu Hatieganu' University of Medicine and Pharmacy, Cluj-Napoca, Romania; ${ }^{3}$ The National Clinical \\ Center for Pediatric Rehabilitation, Bucharest, Romania; ${ }^{4}$ The Medical Service of Metrorex, Bucharest, Romania; ${ }^{5}$ The Ist Clinic \\ Division of Neurosurgery, Emergency Hospital 'Bagdasar-Arseni', Bucharest, Romania; ${ }^{6}$ Midlands Center for Spinal Injuries, \\ Oswestry, UK and ${ }^{7}$ Medical Faculty of the Westfälische Wilhelms University, Münster, Germany
}

Study design: Literature review.

Objectives: To review the main published current neuroprotection research trends and results in spinal cord injury $(\mathrm{SCl})$.

Setting: This paper is the result of a collaboration between a group of European scientists.

Methods: Recent studies, especially in genetic, immune, histochemical and bio (nano)-technological fields, have provided new insight into the cellular and molecular mechanisms occurring within the central nervous system (NS), including SCls. As a consequence, a new spectrum of therapies aiming to antagonize the 'secondary injury' pathways (that is, to provide neuroprotection) and also to repair such classically irreparable structures is emerging. We reviewed the most significant published works related to such novel, but not yet entirely validated, clinical practice therapies.

Results: There have been identified many molecules, primarily expressed by heterogenous glial and neural subpopulations of cells, which are directly or indirectly critical for tissue damaging/sparing/ re-growth inhibiting, angiogenesis and neural plasticity, and also various substances/energy vectors with regenerative properties, such as MAG (myelin-associated glycoprotein), Omgp (oligodendrocyte myelin glycoprotein), KDI (synthetic: Lysine-Asparagine-Isoleucine ' $\gamma$-1 of Laminin Kainat Domain'), Nogo (Neurite outgrowth inhibitor), NgR (Nogo protein Receptor), the Rho signaling pathway (superfamily of 'Rho-dopsin gene-including neurotransmitter-receptors'), EphA4 (Ephrine), GFAP (Glial Fibrillary Acidic Protein), different subtypes of serotonergic and glutamatergic receptors, antigens, antibodies, immune modulators, adhesion molecules, scavengers, neurotrophic factors, enzymes, hormones, collagen scar inhibitors, remyelinating agents and neurogenetic/plasticity inducers, all aiming to preserve/re-establish the morphology and functional connections across the lesion site. Accordingly, modern research and experimental $\mathrm{SCl}$ therapies focus on several intricate, rather overlapping, therapeutic objectives and means, such as neuroprotective, neurotrophic, neurorestorative, neuroreparative, neuroregenerative, neuro(re)constructive and neurogenetic interventions.

Conclusion: The first three of these therapeutical directions are generically assimilated as neuroprotective, and are synthetically presented and commented in this paper in an attempt to conceptually systematize them; thus, the aim of this article is, by emphasizing the state-of-the art in the domain, to optimize theoretical support in selecting the most effective pharmacological and physical interventions for preventing, as much as possible, paralysis, and for maximizing recovery chances after $\mathrm{SCl}$.

Spinal Cord (2009) 47, 716-726; doi:10.1038/sc.2009.52; published online 14 July 2009

Keywords: SCl; neuroprotection; neuroregeneration; recovery

\section{Introduction}

There are at least 330000 people living with spinal cord injury (SCI) sequels in the European Union, with about

Correspondence: Professor G Onose, The Clinic Division of Physical \& Rehabilitation Medicine of the Emergency Hospital 'Bagdasar-Arseni', Berceni Av. no.12, 041915 Bucharest, Romania.

E-mail: geluonose@clicknet.ro

Received 29 August 2008; revised 12 March 2009; accepted 4 April 2009; published online 14 July 2009
11000 new cases every year. ${ }^{1}$ In the United States of America, the number of people living with SCI sequels has been estimated (most recently in 2007) to be between 227080 and 300938 , with an annual incidence of approximately 12000 new cases. ${ }^{2}$

Life expectancy after SCI is longer now than it was a few decades ago, because of improvements in medical and surgical care. Yet, little progress has been made toward 
improving its neurological outcome. Moreover, as it happens for most of the 'chronically' unsolved problems, there seems to be, in the related global mentality, a kind of decrease in interest; there have not been any overall incidence studies on SCI in the United States since the 1970s, hence it cannot be known precisely whether incidence has changed lately. ${ }^{2}$

Most traumatic SCIs do not involve a physical transection of the spinal cord. The lesion is rather the result of a contusive, compressive or stretch injury. In addition to initial tissue damage, much of the post-traumatic degeneration of the cord is due to a multifactorial 'secondary injury process', which takes place during the first minutes, hours, days or even months after the initial trauma; its mechanisms are intricate and not completely understood.

The mechanisms of 'primary injury' themselves are not yet completely elucidated. The contusive model is the most frequent. From a morphological point of view, the 'epicenter' of the SCI is represented by a central hemorrhagic necrosis, surrounded by surviving axons with a centrifugal distribution. There are two paradigms that describe a primary axonal injury. Probably the initial loss of axons has a centrifugal pattern, explicable by a longitudinal displacement of the cord's central content; this is assimilated to the mechanical events occurring in a compressed toothpaste tube (Figure 1).
In addition, the axiom 'a chain is as strong as its weakest link' may explain the vulnerability of large, myelinated axons to trauma; the myelinated portions of these axons are stiff and do not stretch well (although they are generally quite distensible and can elongate more than twice their normal length without breaking - but only when slowly stretched, at less than $0.5 \mathrm{~m} \mathrm{~s}^{-1}$ ). The critical velocity of tissue movement, which will lead to an axonal tear in a spinal cord contusion, is $0.5-1 \mathrm{~m} \mathrm{~s}^{-1}$. The distribution of tissue velocities in the rostral and caudal directions concentrates most of the stretching and shearing forces in the central part of the spinal cord, where the greatest disruptions occur. Almost all of the stretch and shear forces concentrate on the nodes of Ranvier - the weakest link - which can easily break down. Severed axons tend to retract, forming club endings. ${ }^{3}$

The secondary injury process (including the excessive synthesis of nitric oxide and oxidative stress, microglia activation, local inflammation, disturbance of microcirculation, blood-brain barrier dysfunction and the more recently acknowledged 'delayed mechanisms of cell death' $)^{4,5}$ leads to disastrous consequences:

- Neuronal necrosis

- Neuronal apoptosis

- Scar and cyst formation



Figure 1 An attempt to figure out the 'toothpaste' theory with regard to the mechanical morphometric early damages in spinal cord injury (SCI). 
- Demyelination

- Disruption of morpho-functional nerve pathways (disconnection)

The main aim of neuroprotection is to counteract the mechanisms of secondary injury and to minimize their pathological consequences.

The strict theoretical delimitation between neuroprotection and neuroregeneration does not always reflect reality, because a neuroprotective drug/procedure may also facilitate or promote neuroregeneration; a substance that prevents scar formation may be considered neuroprotective, although it also facilitates-even indirectly-regeneration. From a clinical point of view, the therapeutic measures taken to prevent/minimize secondary injury during the acute phase of an SCI may all be considered neuroprotective.

\section{Classification}

Our team has substantial experience in dealing with both spinal cord and brain injuries.
The cascade of pathophysiological events that leads to secondary damage is quite similar in the traumatic injuries of both the brain and the spinal cord; therefore, the objectives and therapeutical means are rather homogenous/overlapping.

Below, we present a relatively complete list of drugs and procedures we reviewed as neuroprotective in spinal cord and brain injuries, within a 'color code', illustrated as follows:

- mainly researched/(relatively) effective in SCI

- rather equally researched/(relatively) effective in SCI and brain injuries

- mainly researched/(relatively) effective in brain injuries

Taking into account a reasonable editorial space, our color code serves to have a balanced, selective approach; the present paper refers to the drugs and procedures mainly researched/(relatively) effective in SCI and to some rather equally researched/(relatively) effective drugs in both SCI and brain injuries. In addition, considering both conciseness and a selective review of recent

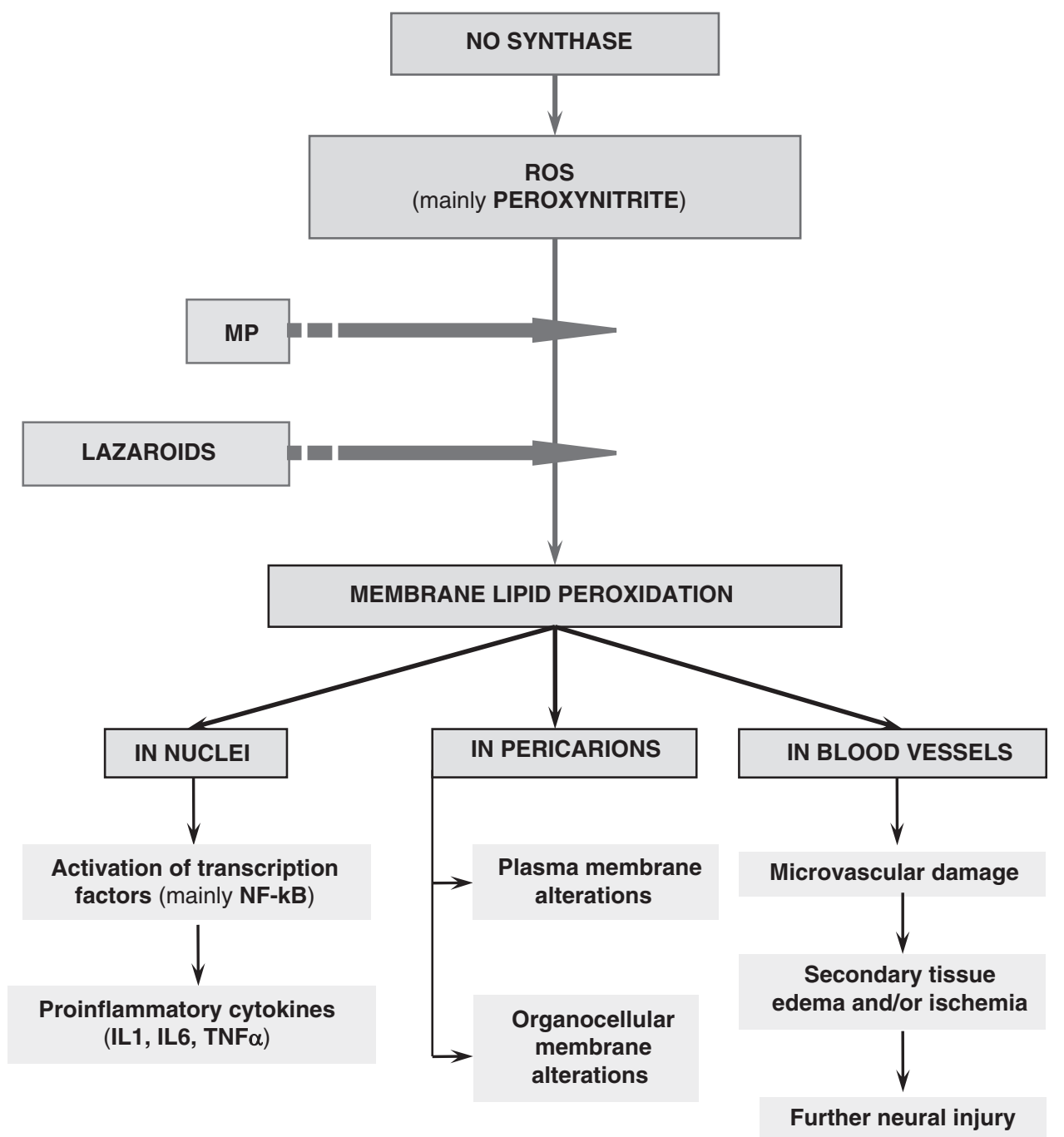

Figure 2 The main protective effects of methylprednisolone (MP), Lazaroids in acute SCI. 
and/or less-discussed matters, not all items will be discussed:

\section{Selective descriptions and discussions}

'Classical' drugs with a long history of clinical use

- Anti-inflammatory drugs and immune modulators:

- SAIDs (steroidal anti-inflammatory drug) (Methylprednisolne) (Figure 2)

- Lazaroids (21-aminosteroids) (Figure 2)

- NSAIDs (non-steroidal anti-inflammatory drug) $\left(\mathrm{COX}_{2}\right.$ inhibitors)

Interleukin 10 (IL-10)

Glatiramer acetate

- Vitamins and other nutritional supplements (B vitamins, tioctic acid, selenium, zinc, magnesium, etc.)

- Iron chelators (deferoxamine, 2,2'-bipyridine, quercetin)

- Lithium

- $\mathrm{Ca}^{2+}$ channel antagonists

- Peptide mixtures with neurotrophic actions (Cerebrolysin, Actovegin)

- Musculotropic vasodilators

- Nootropic agents

- Anesthetic agents (including Xenon gas, with anti-excitotoxic properties)

- (Other) hormones

- 17-estradiol, progesterone

- TRH (thyrotropin-releasing hormone) and analogs

Statins (Simvastatin) - acting as antagonists of growth inhibitory signals in central nervous system (CNS)

- Anti-excitotoxic agents (glutamate blockers): ionotropic receptor-NMDA ( $N$-methyl-D-aspartic acid), AMPA $(\alpha-$ amino-3-hydroxyl-5-methyl-4-isoxazole-propionate)

blockers; metabotropic receptor-kainate-blockers.

\section{New/experimental drugs/procedures}

- KDI tripeptide

- Antagonists of growth inhibitory signals in CNS

- Monoclonal antibodies to Nogo

Phosphodiesterase inhibitors (Rolipram)

Dibutyril cyclic AMP (db cAMP) Inhibitors of Rho signaling (Cethrin)

- Scar-preventing substances (chondroitinase ABC, cordaneurin, EphA4 antagonists)

- Immune therapy with activated macrophages

- Monosialoganglioside GM 1

- IGF-1 (insulin-like growth factor)

- Neurotrophins-additionally characterized by their pleiotropic effects ${ }^{6}$

- NGF (nerve growth factor)

NT-3 (neurotrophin-3)

BDNF (brain-derived neurotrophic factor)

GDNF (glial-derived neurotrophic factor)

CNTF (ciliary neurotrophic factor)

- Antioxidants

EAAC1 protein
- Free radical scavengers: ascorbate, vitamin E, betacarotene, alpha-tocopherol, penicillamine, superoxide dismutase, Q10 coenzyme

L-cysteine

- Apoptosis inhibitors

- Protease inhibitors (caspase inhibitors, calpain inhibitors)

- PARP (poly (ADP-ribose) polymerase) inhibitors

- Cell adhesion molecules (L1-CAM)

- DMSO (dimethyl sulfoxide)

- Erythropoietin

- Melatonin

- Inosine (Axosine)

- AIT-082 (leteprinim potassium, Neotrofin)

- 4-aminopyridine (Fampridine)

- Riluzole

- Fusion technology (polyethylene glycol)

- Physical therapy

- Low-level LASER (light amplification by stimulated emission of radiation) therapy

- Oscillating field stimulator

- Functional electrical stimulation

Repetitive transcranial magnetic stimulation

- Pharmacological and physiatric stimulation of the spinal central pattern generator (CPG)

- Local hypothermia

- Hyperbaric oxygen therapy

- Combinatory ('COMBO') strategies

\section{'Classical' drugs with a long history of clinical use}

\section{Lithium}

Recent studies have tested intrathecally administered lithium for SCI ( $4.24 \mathrm{ml}$ per injection, above and below the spinal cord lesion), as well as its use in conjunction with neonatal umbilical cord blood mononuclear cells. These studies and preliminary clinical tests with oral lithium on small numbers of volunteers with acute and chronic SCI came with not impressive, but yet promising results. ${ }^{7} \mathrm{~A}$ phase II clinical trial designed to test the safety and efficacy of lithium carbonate in chronic SCI is currently ongoing. ${ }^{8}$

\section{New/experimental drugs and procedures}

The KDI tripeptide domain of $\gamma 1$-chain of laminin This is a synthetic tripeptide (Lys-Asp-Ile) ${ }^{9}$ that has been studied during the past few years for its neuroprotective and neurite outgrowth-promoting properties.

Experiments on human embryonic neocortical neurons indicated that this tripeptide is a universal and potent inhibitor of NMDA, AMPA and of kainate subclasses of glutamate receptors, with a non-competitive action on AMPA receptor channel activity. ${ }^{10}$

The tripeptide was identified by researches at the University of Helsinki, Finland and at the Johnnie B. Byrd SR Alzheimer's Center and Research Institute. They found that KDI facilitated axon guidance, modulated electrical activity and promoted the survival of rat hippocampal neurons. 
Subsequent experimental studies showed that, in cultured cells, the biologically active KDI domain selectively attracted the directional outgrowth of neurites from explants of the dorsal spinal cord. ${ }^{11}$

Recent studies highlighted another potential neuroprotective mechanism for the KDI tripeptide: it seems to neutralize both glial- and myelin-derived inhibitory signals that physiologically limit the regeneration processes in CNS. ${ }^{12}$ The studies on rats showed no significant side effects. ${ }^{13}$

So far, preclinical and clinical researches on glutamate blockers have been focused on stroke and chronic neurological disorders, hence data about their efficiency in SCI are scarce. KDI is the only compound that has been thoroughly tested on SCI, but never in a clinical trial.

\section{Antagonists of the growth inhibitory signals in CNS}

Regeneration of injured neurons is severely limited in adult mammalian CNS. The environment of the lesioned spinal cord embarrasses regeneration owing to the presence of inhibitory myelin-derived proteins and to the formation of an astroglia scar. Although the mechanisms are still unclear, there are different classes of inhibitory molecules that physiologically prevent nerves from regrowing after injury, mainly by binding to specific receptors on the axon growth cone. Theoretically, molecules that block such inhibitors may enhance axonal regeneration and functional recovery.

To date, there are three myelin proteins known to inhibit axonal outgrowth: Nogo, MAG and OMgp. They are generically called axon regeneration inhibitors.

M Schwab, from the University of Zurich, Switzerland, was the first to identify a myelin-associated neurite outgrowth inhibitor, a protein he called Nogo-A. The activation of its receptor results in a decrease in cellular cAMP. ${ }^{14}$ Schwab's team designed neutralizing antibodies to this protein, which promoted neuronal growth in cell cultures. ${ }^{15}$

Anti-Nogo antibodies: $11 C 7$ and 7B12. The experiments of Schwab's team showed that, if infused into the damaged spinal cord of rats, the $11 \mathrm{C} 7$ and 7B12 compounds could trigger axonal regeneration with an impressive functional recovery. Histology showed long-distance regeneration and sprouting in the corticospinal tract, actually bridging the lesioned areas. The antibodies did not cause hyperalgesia, which may indicate that neuronal rewiring took place in a correct manner. ${ }^{16}$ In fact, the same team had earlier studied genetically engineered Nogo-A knockout mice and found no evidence of an abnormal development of CNS, or of any other side effects. The lack of anomalies in these Nogo-Adeficient animals is a solid argument for the safety of the therapy with Nogo-A antibodies. ${ }^{17}$

Further testing on monkey models with SCI proved antiNogo-A antibodies to be effective and safe. ${ }^{18,19}$ These results encouraged scientists to start human tests. They are currently recruiting patients for a phase I clinical trial.

MAG antagonists. Myelin-associated glycoprotein is a member of the Siglec family of sialic acid-binding lectins, which binds to sialoglycoconjugates on axons, and particularly to gangliosides, GD1a and GT1b. During the past decade, researchers identified several monovalent sialoside inhibitors of MAG. ${ }^{20}$ A phase I clinical trial with anti-MAG antibodies for the therapy of SCI started in 2007 and is currently ongoing. ${ }^{8}$

Sialidase. Researchers from Johns Hopkins University, Baltimore and from the University of Michigan, Ann Arbor, USA, have been testing sialidase, an enzyme that cleaves one class of axonal MAG receptors. At 4 weeks after administration of Clostridium perfringens sialidase into a transplanted nerve, injected to rejoin avulsed nerve endings, it revealed 2.6-fold the amount of nerve regeneration compared with control. Chondroitinase $\mathrm{ABC}$ also enhanced the axon outgrowth in this model. ${ }^{21}$

Rolipram. A major effect of myelin-associated inhibitory proteins is the decrease of cellular cAMP levels. cAMP depletion is a contributing factor to secondary tissue damage and many research efforts are focused on antagonizing this effect.

Rolipram inhibits phosphodiesterase 4, thereby raising the level of cAMP. It was used in the past as an antidepressant, but in oral administration it had severe adverse reactions, such as nausea and vomiting. As a consequence, it was withdrawn from psychiatric use. Rolipram readily crosses the blood-brain barrier; for the treatment of SCI, the drug can be used in injectable form, hence gastrointestinal side effects should not be a problem. Besides, Rolipram may need to be given only for a short period after SCI.

Animal tests with Rolipram showed that it enhanced neural regeneration and diminished apoptosis in SCI models, ${ }^{22}$ improving the functional outcome. There was also an attenuation of reactive gliosis. ${ }^{23}$

Rolipram recently underwent a clinical trial for multiple sclerosis, ${ }^{8}$ but so far it has not been clinically tested for SCI.

Dibutyril cAMP. This is a synthetic analog of cAMP, designed to cross cellular membranes. Preclinical studies showed that db-cAMP has the same effects as Rolipram. ${ }^{24}$

\section{Inhibitors of rho signaling (Cethrin)}

Many studies indicated that NgR signals to Rho GTPase, the intracellular messenger that mediates the effects of axonal growth inhibitors. ${ }^{25}$

Many compounds have been tested in vitro and in vivo to inactivate the Rho pathway, and results showed that they promoted neurite outgrowth. Currently, only one of these compounds is undergoing clinical trials (Cethrin). ${ }^{8}$

Cethrin (BA-210) is a recombinant protein-based inhibitor of Rho signaling. It combines a biochemical analog of a bacterial toxin, Rho GTPase antagonist, with a fibrin sealant to optimize its delivery during spinal surgery. Preclinical studies showed that it has good neuroprotective and neuroregenerative effects on injured spinal cord neurons and showed no evidence of adverse effects. ${ }^{25}$

In 2005, the USA Food and Drug Administration approved an orphan drug status for Cethrin for the treatment of acute 
thoracic and cervical SCI, and subsequently, the producer initiated a phase I/IIa multicenter, dose-escalating, safety and efficacy clinical trial of Cethrin in acute SCI. As the preliminary results of this trial seemed encouraging (significant motor improvement and no serious side effects, according to a company press release), the drug is currently being tested in a phase IIb multicenter clinical trial (assessing the safety and efficacy of intraoperative epidural Cethrin in adults with acute cervical SCI). ${ }^{8}$

\section{Scar-preventing substances}

Spinal cord injury is followed by the formation of a glial scar at the lesion site. This scar is not only a physical but also a biochemical/signaling barrier for neural regeneration.

Chondroitin sulfate proteoglycans, which are synthesized by injury-activated astroglia and deposited at the damaged site, have been identified as signals that restrict regeneration and plasticity.

Chondroitinase $\mathrm{ABC}$ is a bacterial enzyme that catalyzes the hydrolysis of the chondroitin chains of proteoglycans, thus achieving not only the blockade of the inhibitory signals of chondroitin sulfate proteoglycans but also the partial removal of the physical barrier they form.

A continuous delivery of chondroitinase $\mathrm{ABC}$ into the injury site was reported to induce axonal elongation and substantial functional recovery in murine models of acute SCI. ${ }^{26}$ Furthermore, a study on rats showed that chondroitinase is effective in promoting both somatic and autonomic motor recovery after a spinal cord contusion injury. ${ }^{27}$ The clinical use of chondroitinase may be set back by two major impediments: its synthesis is still very expensive and, on the other hand, being of nonhuman origin (Escherichia coli), chondroitinase ABC may carry immunological risks.

Cordaneurin is a drug developed in Germany to prevent collagen scar formation; it has been successfully tested in preclinical trials and, in 2004, was granted orphan drug designation for traumatic SCI by the European Medicines Agency. In 2005, it entered clinical trials. The clinical application of Cordaneurin comprises a neurosurgical intervention that must be performed during the first $3 \mathrm{~h}$ of initial trauma. $^{28}$

Ephrin A4 antagonists. Ephrins form a large subfamily of receptor tyrosine kinases that are implicated in cell-cell interactions. Their roles in the embryonic morphogenesis and postnatal development of CNS are largely accepted, although not completely understood. To date, 14 distinct Eph receptors have been identified, one among them being EphA4.

A research team at the University of Melbourne, Australia studied mice lacking the EphA4 gene. After spinal cord hemisection, histology showed that axons formed multiple pathways, crossing the lesion site. The EphA4 expression on astrocytes was upregulated at the injury site in wild-type mice, whereas EphA $4^{-1-}$ mice had less gliosis and significantly reduced glial scars in their lesioned cords. The neurological outcome (assessed within 1-3 months of injury) was dramatically improved in EphA $4^{-/-}$mice. ${ }^{29}$ The study led to the conclusion that EphA4 is not only responsible for astrocyte activation and scar formation but also for axonal inhibition.

\section{Apoptosis inhibitors}

After SCI, apoptosis occurs in parallel with necrosis, and a delayed apoptosis significantly contributes to secondary damage. Necrosis is more prominent after severe insults, whereas apoptosis is triggered by milder injury ${ }^{30}$ and evolves over a longer period of time. Studies on SCI animal models showed that apoptosis is a process that begins within the first hours of injury and continues for as long as (mainly) 3 weeks. Apoptosis in glial cells is especially prolonged, inflicting an important loss of oligodendrocytes, which in turn leads to extensive demyelination weeks after the initial lesion. These facts are important in defining the therapeutic window for the potential use of apoptosis inhibitors.

In experimental conditions, blocking various apoptosis pathways, either by pharmacological agents or by genetic manipulation, yielded encouraging results.

Caspase inhibitors. Caspases are synthetized as inactive precursors; when activated, they initiate a death program by destroying key cellular substrates and by activating factors that mediate further damage. Caspases are classified into upstream initiators and downstream executioners. Upstream caspases are activated by a cell-death signal. ${ }^{30,31}$ In turn, they activate downstream caspases, which directly mediate the events leading to cellular death. ${ }^{30,32}$ The final phase of the process begins when the executioner caspases activate the nucleases that degrade DNA. ${ }^{30}$

Experimental studies showed that caspase activity significantly increases in the spinal cord after traumatic injuries. ${ }^{32}$ Treating lab animals with caspase inhibitors (caspase-9 inhibitor z-LEHD-fmk) seemed to reduce the morphological changes in the traumatized spinal cord and improved the functional outcome in some studies, ${ }^{33}$ whereas other researchers found no benefits with the use of caspase inhibitors. $^{34}$

Calpain inhibitors. Calpains are a large family of $\mathrm{Ca} 2+-$ dependent cysteine proteases. Their function is not completely elucidated, but a number of their substrates have already been identified. Calpains cleave many cytoskeletal and myelin proteins, along with growth factor receptors, transcription factors and cell-cycle-related proteins. ${ }^{35}$ Depending on tissue distribution, calpains are classified as ubiquitous and tissue-specific. Ubiquitous isoforms are abundantly expressed in CNS and their activity is regulated by calpastatin, an endogenous protein inhibitor. ${ }^{35}$

The increase in intracellular $\mathrm{Ca} 2+$ levels that takes place after an SCI overactivates calpains. Calpastatin may be degraded by calpain overactivation, losing its regulatory activity. An uncontrolled calpain activity results in the degradation of key cytoskeletal, membrane and myelin components, and, in conjunction with caspases, can lead to apoptosis. ${ }^{36}$ 
Several synthetic calpain inhibitors (4-quinolinone 2carboxamides) have been developed and experimental studies on murine models of SCI showed that their administration reduced apoptotic cell death, diminished gliosis and improved neurological outcome. ${ }^{37}$

\section{Erythropoietin}

Erythropoietin stimulates hematopoiesis and also has cytoprotective properties. Studies with recombinant human erythropoietin in animal models of spinal cord traumatic and ischemic injuries showed that, if administered immediately after injury, it inhibits lipid peroxidation and neuronal apoptosis. ${ }^{38}$ However, high doses are required to achieve neuroprotection, and they carry a risk for polycythemia, hyperviscosity syndrome and hypertension.

The carbamylated erythropoietin is a newly developed, distinct isoform that does not bind to the classical erythropoietin receptor and has no hematopoietic properties. ${ }^{39}$ It has been shown to possess neuroprotective effects in animal models of SCI, stroke, diabetic neuropathy and autoimmune encephalomyelitis. $^{40}$

A phase III clinical trial assessing the safety and efficacy of erythropoietin versus methylprednisolone in acute SCI is currently ongoing. ${ }^{8}$

4-Aminopyridine (Fampridine). Fampridine is a relatively selective blocker of voltage-activated potassium channels, which has been studied for its capacity to restore conduction in injured nerves. The known side effects of 4 -aminopyridine include peripheral vasospasm, dizziness, nausea, gastritis, paresthesia and thrombocytopenia. ${ }^{41}$ High doses can cause convulsions.

The drug has an orphan drug status for multiple sclerosis in the United States and has undergone phase III clinical studies for this disease. A recently completed clinical trial showed that oral therapy with sustained-release fampridine (Fampridine-SR) significantly improved walking ability in some multiple sclerosis patients. ${ }^{42}$

The producing company has been testing Fampridine-SR in phase III clinical trials for the treatment of spasticity in incomplete SCI, but so far the reported results have shown that the obtained improvements were not statistically significant. It should be mentioned that earlier reports from clinical trials with smaller numbers of subjects had found more encouraging results with the use of this drug in chronic, incomplete SCI: they had reported significant improvements in motor and sensory functions. ${ }^{41}$

Riluzole. Riluzole is a sodium channel blocker with antiexcitotoxic effects, already registered for the treatment of amiotrophic lateral sclerosis. Experimental studies in rats showed significant improvements of neuronal sparing and reinnervating capacity after SCI. ${ }^{43}$ Yet, although it would seem that they are easier to carry out, considering riluzole is in clinical use, no clinical trials have been conducted for this drug in SCI. ${ }^{44}$

\section{Current physiatric endeavors}

Low-level LASER therapy. Juanita Anders and coworkers (Uniformed Services University, Bethesda, Maryland, USA) ${ }^{45}$ studied the impact of low-power LASER irradiation on animals with SCI, with peripheral nerve injuries and with retinal disorders. This therapy, with an $810 \mathrm{~nm}$-wavelength LASER, stimulated axonal regeneration and improved the neurological outcome in animals with SCI. Low-power LASER also reduced inflammatory reaction during the acute phase.

Functional electrical stimulation (FES). FES has been used for decades in patients with various types of palsies, and seems particularly beneficial in SCIs, in which this therapy is applied to prevent atrophy, to compensate muscle dysfunction, to maintain mobility and therefore to improve the quality of life. In current medical practice, functional electrical stimulation is applied externally (by means of simple electrodes, or, more recently, as a component of hybrid orthotic systems). Modern research is exploring the advantages of an implanted functional electrical stimulation version (neuroprosthesis). A team of researchers at the Medical University Münster, Germany has already used implanted functional electrical stimulation to restore gait (by stimulating a few muscle groups that are essential for locomotion) in paraplegic patients with chronic SCI (more than 1 year after the trauma), and their results are promising. ${ }^{46}$

Oscillating field stimulator (OFS). The application of an oscillating, low-voltage, direct current of electricity, which generates an electrical field mimicking the polarity guidance in the developmental stages of CNS, to the areas above and below an SCI stimulates the surrounding neural fibers to grow across the lesion site.

A team at Purdue University experimented with an electrical field in which polarity was inversed every $15 \mathrm{~min}$ on dogs with acute SCI. After obtaining positive results, they conducted a preliminary phase 1 clinical trial with OFS in patients with complete SCI.

The oscillating field is generated by a battery-powered device that needs to be attached to the bone. The device was implanted within 18 days of injury and explanted at 15 weeks. The results showed a better neurological outcome (in comparison with the control group) at 2 months, 6 months and 1 year. There was one case of wound infection after explantation. ${ }^{47}$ The University obtained permission for enrollment of additional patients, and the device (Andara) is currently the subject of a phase Ib clinical trial. The device is designed to be used at the time of orthopedic stabilization of injury, and is removed after 14 weeks.

Repetitive transcranial magnetic stimulation (rTMS). The principle of this therapy is to non-invasively induce electric fields in the brain, using a powerful, rapidly changing magnetic field. rTMS has been a useful tool in brain mapping and has shown promise in the therapy of Parkinson's disease, stroke, 
depression and hallucinations. The procedure is completely safe and does not produce any discomfort in the patient.

A preliminary clinical trial tested repetitive transcranial magnetic stimulation on patients with incomplete SCIs. It showed that, when applied over the motor cortex, the magnetic stimulation altered the cortical inhibition and improved the motor and sensory function in these patients. $^{48}$

Pharmacological and physiatric stimulation of the spinal central pattern generator (CPG)

The spinal CPG is a new morpho-functional concept that seems extremely promising, offering new perspectives for the therapy and rehabilitation of motor impairments. It consists of a neural network, located in the spinal cord (approximately at the level of L1 and L2), which is capable of endogenously (quasi-independently) generating rhythmic nervous outputs for the pattern of locomotion.

Pharmacological stimulation of spinal CPG. Evidence from in vitro and in vivo studies suggests that serotonin 5-hydroxytryptamine (5-HT) receptors are associated with a massive synaptic amplification within the CPG. A number of studies (unfortunately, most of them performed on mice-but there are significant differences in the organization and activity of spinal CPG in quadrupeds, in comparison with bipeds) got encouraging functional results (restoring active movements in hind limbs after an SCI) with the use of 5-HT alone, or in combination with other monoamines, such as dopamine. ${ }^{49}$ The stimulation of serotonin receptors, which are apparently highly expressed in CPG, elicited stepping with rhythmic but uncoordinated, asynchronous movements. Moreover, recent studies claim the identification of a descendent serotoninergic pathway, implicated in locomotion induction in mammals, through the activation of serotonin receptors in Rexed's lamina 7 of the distal dorsal spinal cord, that is, in the proximity of the CPG. ${ }^{50}$ Another study found that injecting neural stem cells at the T11 level of the injured spinal cord restored locomotion in lab mice. ${ }^{51}$

Experimental data (from studies on animal models, including cats) showed that CPG can also be stimulated by alpha-1 and alpha-2 adrenergic agonists (methoxamine, clonidine), serotoninergic agonists (quipazine) and glycine receptor antagonists (strychnine). ${ }^{52}$ Adrenergic and serotoninergic agonists were used in a study to directly activate spinal CPG through an intrathecal injection in rabbits with incomplete SCI, but their effects lasted only for a short time, during the early post-lesion period. ${ }^{53}$

Physiatric stimulation of spinal CPG. Progressive verticalization basically aims at rendering profitable the residual motor function in the lower limbs. Recent studies provided new insights into the molecular and genetic mechanisms through which an alternate postural load can stimulate CPG activity, namely, stimulation of the Hox genes (essential in the organization/reorganization processes in the CNS -21 Hox proteins are expressed by spinal cord motor neu-



Figure 3 Tilt table.

rons), ${ }^{54,55}$ brain-derived neurotrophic factor ${ }^{56}$ and synapsin 1 release.

Generally, postural load is initially achieved using a tilt table. Passive verticalization (Figure 3) stimulates lumbar CPG, proprioception/sensory-motor feedback, postural reflexes, ${ }^{57}$ as well as bone trophicity, urine drainage and intestinal transit, and, last but not the least, it enhances selfesteem and quality of life.

Later in the rehabilitation process, an alternate load can be achieved by using standing frames (Figure 4); wheelchairs with verticalization facilities are more efficient and modern devices that can be employed to the same end.

For patients with incomplete tetra/paraplegia, as well as for hemiplegics, progressive verticalization and exercising alternate postural load may prove to be even more efficient than the conventional methods of proprioceptive facilitation. ${ }^{58}$

Some authors support the hypothesis that treadmill training (gait exercise) stimulates CPG activity, facilitates the reactivation of cortical engrams for gait patterns and may also induce axonal growth and synapse formation in the spinal cord, even below the injury level. ${ }^{59}$ A new technique, body-weight-supported treadmill training, was developed, using a device that allows the therapist to control weight bearing and speed during the exercise. An important advantage of this method versus conventional treadmill training is the offloading mechanism. Preliminary clinical studies came up with good functional results in patients with incomplete SCI. ${ }^{60}$ 


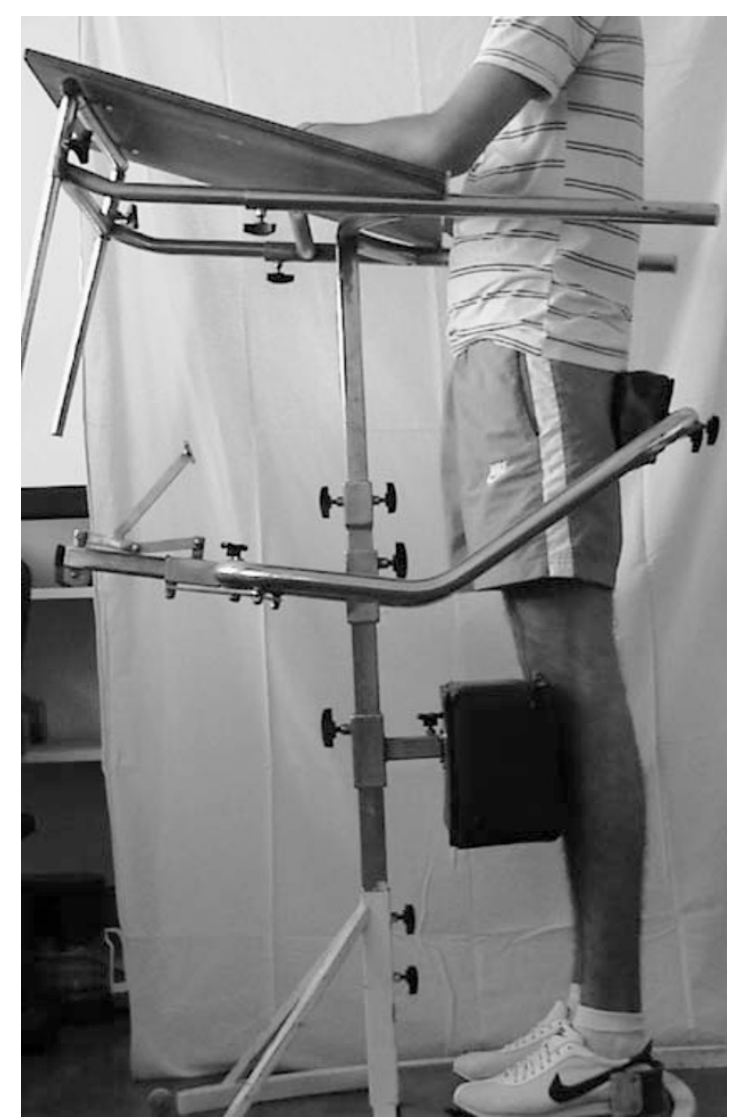

Figure 4 Standing frame.

Epidural electrical stimulation is a method that combines physical therapy and neurosurgery. It may provide a new therapeutic approach to complete SCI, as well as objective, electrophysiological proofs for the autonomous activity of lumbar CPG. Studies on both lab animals ${ }^{61}$ and humans (a total of 10 patients) showed that low frequency electrical stimulation $(2.2-50 \mathrm{~Hz})$ with epidural electrodes elicited stepping-like movements; although a synergistic activation of kinematic chains (triple flexions alternating with extensions) was observed, it is obvious that a functional gait could not be achieved. ${ }^{62,63}$ It should be noted that this technique involves some significant risks for the patient, such as infections, local hemorrhages, etc.

\section{Autologous nerve bypass grafts}

In patients with complete paraplegia after SCI, an experimental updating surgical procedure has been applied to bypass the lesion site and reconnect the corticospinal tract with the main standing/walking muscles of the legs, using the sciatic nerve. ${ }^{64}$ Such an intervention was performed by a German research team in a young woman with a complete T9 lesion, and the first voluntary movements of the connected muscles were noticed 17 months later; gait with the help of a walker became possible after 27 months. ${ }^{65}$ These results are very promising, yet further studies are necessary.

\section{Combinatory strategies}

Combinatory strategies using a regenerative procedure (such as stem cells) together with neuroprotective pharmaceuticals provide a multi-targeted approach in SCIs. Several researcher teams have been using Schwann or olfactory ensheathing glia cell grafts in combination with neuroprotective factors, to provide a more favorable microenvironment. Below are a few examples of the most illustrative of such strategies.

Researchers at the Miami Project used Rolipram and db cAMP in combination with a Schwann-cells transplant into the injury site. The therapy was designed in three steps: first, Rolipram was administered subcutaneously at the time of injury; 1 week later, Schwann cells were injected into the injury site; and then db cAMP was injected above and below the graft. The treated animals achieved an impressive functional recovery after SCI. Furthermore, histology showed that a large number of axons grew into the graft end, even past the lesion site. ${ }^{66}$

The combination between Schwann cell bridges, olfactory ensheathing glia grafts and chondroitinase was tested in animals with an acute transection of the spinal cord. This therapy increased the number of myelinated axons in the Schwann bridge and of serotonergic fibers that grew through the bridge, in comparison with grafts only or with untreated groups. $^{67}$

A study combined a peripheral nerve graft with chondroitinase to treat rats with an acute hemisection of the spinal cord. The treated animals showed a better functional outcome compared with that in the graft-only group. In addition, histology showed that more axons extended for longer distances than in the graft-only group. ${ }^{68}$

As shown even in the abstract, modern approaches to CNS injuries consist of a continuous endeavor that should be started as close as possible to the initial lesion and pursued until the chronic stage in the form of pathogenic (hopefully, in the near future, as basic, curative) therapy, comprising seven main directions, from neuroprotection, the earliest, to neurogenetic interventions. The first three among them belong to an extended area of neuroprotection, including pleiotropic effect maximization, which represents the focus of this review. ${ }^{6,7,44}$ But, obviously, the wide spectrum of post-SCI pathology (especially in patients with a complete cervical SCI, which can be considered 'hyperchronic') ${ }^{6}$ needs practically lifelong prevention, care, therapy and rehabilitation, including family/social/professional reinsertion and targeted on each possible symptom/complication (for example: pressure sores, spasticity, sexual dysfunctions, infertility, incontinence, autonomic dysreflexia, orthostatic hypotension, respiratory/metabolic/gastrointestinal complications, osteoporosis, heterotopic ossification, etc.).

\section{Conclusions}

Neuroprotection is a vast and dynamic field of research. Understanding the intimate mechanisms of secondary injury after CNS trauma provides us with a large number of therapeutic targets. 
A growing number of potential therapeutic interventions in SCI are being clinically tested. Yet most of them are still in the preliminary phases of assessment. To date, only six drugs have been tested in phase III clinical trials, with published results (methylprednisolone, naloxone, trilazad mesylate, monosialoganglioside GM1, 4-aminopyridine and tizanidine). Methylprednisolone was the only one to have proven some (modest) beneficial effects in acute SCI. Although clinical trials have been few in SCI, and came with frustrating results, some promising therapeutic tools (such as lazaroids, lithium and IL-10) have not yet been appropriately tested. Apart from combinatory strategies, at present, we consider the following to be the most promising among the ongoing monotherapy neuroprotective endeavors (obviously, each of them integrable in combinatory paradigms): anti-Nogo antibodies, carbamylated erythropoietin, Cethrin, lithium and OFS.

\section{References}

1 http://www.travisroyfoundation.org/pages/resources-stats.htm

2 http://www.spinalcord.uab.edu/show.asp?durki=116979\&site $=1021 \&$ return=19775.

3 Blight AR, Decrescito V. Morphometric analysis of experimental spinal cord injury in the cat; the relation of injury intensity to survival of myelinated axons. Neuroscience 1986; 19: 321-341.

4 Fehlings MG, Baptiste DC. Current status of clinical trials for acute spinal cord injury. Injury 2005; 36(Suppl 2): B113-B122.

5 Hall ED, Springer JE. Neuroprotection and acute spinal cord injury: a reappraisal. NeuroRx 2004; 1: 80-100.

6 Mureşanu D. Neuroprotection and Neuroplasticity-Two Aspects of a Continuous Process, Genetically Regulated and Powered by Neurotrophic Factors. The 6th AMN Congress. Cluj-Napoca, Romania, 2008.

7 Young W. Collaborative neurosciences and biological therapy of spinal cord injured people. Summer School for the Biological Treatment of Chronic Spinal Cord Injury; October 2008; Vienna, Austria, 2008.

8 http://clinicaltrials.gov.

9 http://www.chem.qmul.ac.uk/iupac/AminoAcid.

10 Moykkinen T, Liebkind R, Sjoberg J, Korpi Esa R, Liesi P. The neuroprotective KDI domain of $\gamma 1$ laminin is a universal and potent inhibitor of ionotropic glutamate receptors. J Neurosci Res 2005; 81: 797-804.

11 Liesi P, Laatikainen T, Wright JM. Biologically active sequence (KDI) mediates the neurite outgrowth function of the gamma-1 chain of laminin-1. J Neurosci Res 2001; 66: 1047-1053.

12 Liebkind R, Laatikainen T, Liesi P. Is the soluble KDI domain $\gamma 1$ laminin a regeneration factor for the mammalian central nervous system? J Neurosci Res 2003; 73: 637-643.

13 Wiksten M, Vaananen AJ, Liebkind R, Liesi P. Regeneration of adult rat spinal cord is promoted by the soluble KDI domain of $\gamma 1$ laminin. J Neurosci Res 2004; 78: 403-410.

14 Kartje GL, Schulz MK, Lopez-Yunez A, Schnell L, Schwab ME. Corticostriatal plasticity is restricted by myelin-associated neurite growth inhibitors in the adult rat. Ann Neurol 1999; 45: 778-786.

15 Buchli AD, Schwab ME. Inhibition of Nogo: a key strategy to increase regeneration, plasticity and functional recovery of the lesioned central nervous system. Ann Med 2005; 37: 556-567.

16 Weinmann O, Schnell L, Ghosh A, Montani L, Wiessner C, Wannier $\mathrm{T}$ et al. Intrathecally infused antibodies against Nogo-A penetrate the CNS and downregulate the endogenous neurite growth inhibitor Nogo-A. Moll Cell Neurosci 2006; 32: 161-173.

17 Simonen M, Pedersen V, Weinmann O, Schnell L, Buss A, Ledermann B et al. Systemic deletion of the myelin-associated outgrowth inhibitor Nogo-A improves regenerative and plastic responses after spinal cord injury. Neuron 2003; 38: 201-211.
18 Fouad K, Klusman I, Schwab ME. Regenerating corticospinal fibers in the Marmoset (Callitrix jacchus) after spinal cord lesion and treatment with the anti-Nogo-A antibody IN-1. Eur J Neurosci 2004; 20: 2479-2482.

19 Freund P, Wannier T, Schmidlin E, Bloch J, Mir A, Schwab ME et al. Anti-Nogo-A antibody treatment enhances sprouting of corticospinal axons rostral to a unilateral cervical spinal cord lesion in adult macaque monkey. J Comp Neurol 2007; 502: 644-659.

20 Vyas AA, Blixt O, Paulson JC, Schnaar RL. Potent glycan inhibitors of myelin-associated glycoprotein enhance axon outgrowth in vitro. J Biol Chem 2005; 280: 16305-16310.

21 Yang LJ, Lorenzini I, Vajn K, Mounterey A, Schraam LP, Schnaar RL. Sialidase enhances spinal axon outgrowth in vivo. Proc Natl Acad Sci USA 2006; 103: 11057-11062.

22 Whitaker CM, Beaumont E, Wells MJ, Magnuson DS, Hetman M, Onifer SM. Rolipram attenuates acute oligodendrocyte death in the adult rat ventrolateral funiculus following contusive cervical spinal cord injury. Neurosci Lett 2008; 438: 200-204.

23 Nikulina E, Tidwell JL, Dai HN, Bregman Barbara S, Filbin Marie T. The phosphodiesterase inhibitor rolipram delivered after a spinal cord lesion promotes axonal regeneration and functional recovery. Proc Natl Acad Sci USA 2004; 101: 8786-8791.

24 Qiu J, Cai D, Dai H, McAtee M, Hoffman PN, Bregman BS et al. Spinal axon regeneration induced by elevation of cyclic AMP. Neuron 2002; 34: 895-903.

25 McKerracher L, Winton MJ. Targeting Rho to stimulate repair after spinal cord injury. Top Spinal Cord Inj Rehabil 2003; 8: 69-75.

26 Bradbury EJ, Moon LD, Popat RJ, King VR, Bennett GS, Patel PN et al. Chondroitinase $\mathrm{ABC}$ promotes functional recovery after spinal cord injury. Nature 2002; 416: 636-640.

27 Caggiano AO, Zimber MP, Ganguly A, Blight AR, Gruskin EA. Chondroitinase $\mathrm{ABC}$ improves locomotion and bladder function following contusion injury of the rat spinal cord. J Neurotrauma 2005; 22: 226-239.

28 www.neuraxo.com/cordaneurin.0.html? \&L=2.

29 Goldshmit Y, Galea MP, Wise G, Bartlett PF, Turnley AM. Axonal regeneration and lack of astrocytic gliosis in EphA4-deficient mice. J Neurosci 2004; 24: 10064-10073.

30 Friedlander RM. Apoptosis and caspases in neurodegenerative diseases. N Engl J Med 2003; 348: 1365-1375.

31 Hengartner MO. The biochemistry of apoptosis. Nature 2000; 407: 770-776.

32 Shi Y. Mechanisms of caspase activation and inhibition during apoptosis. Moll Cell 2002; 9: 459-470.

33 Colak A, Karaoglan A, Barut S, Kokturk S, Akyildiz AI, Tasyurekli M. Neuroprotection and functional recovery after application of the caspase-9 inhibitor z-LEHD-fmk in a rat model of traumatic spinal cord injury. I Neurosurg Spine 2005; 2: 327-334.

34 Ozawa H, Keane RW, Marcillo AE, Diaz PH, Dietrich WD. Therapeutic strategies targeting caspase inhibition following spinal cord injury in rats. Exp Neurol 2002; 177: 306-313.

35 Yakovlev AG, Faden AI. Mechanisms of neural cell death: implications for development of neuroprotective treatment strategies. NeuroRx 2004; 1: 5-16.

36 Sribnick EA, Matzelle DD, Banik NL, Ray SK. Direct evidence for calpain involvement in apoptotic death of neurons in spinal cord injury in rats and neuroprotection with calpain inhibitor. Neurochem Res 2007; 32: 2210-2216.

37 Nam DH, Lee KS, Kim SH, Kim SM, Jung SY, Chung SH et al. Design and synthesis of 4-quinolinone 2-carboxamides as calpain inhibitors. Bioorg Med Chem Lett 2008; 18: 205-209.

38 Kontogeorgakos VA, Voulgaris S, Korompilias AV, Vekris M, Polyzoidis KS, Bourantas K et al. The efficacy of erythropoietin on acute spinal cord injury. An experimental study on a rat model. Arch Orthop Trauma Surg 2009; 129: 189-194.

39 Coleman TR, Wetenfelder C, Togel FE, Yang Y, Hu Z, Swenson L et al. Cytoprotective doses of erythropoietin or carbamylated erythropoietin have markedly different procoagulant and vasoactive activities. Proc Natl Acad Sci USA 2006; 103: 5965-5970. 
40 Lapchak PA. Carbamylated erythropoietin to treat neuronal injury: new development strategies. Expert Opin Investig Drugs 2008; 17: 1175-1186.

41 Grijalva I, Guízar-Sahagún G, Castañeda-Hernández G, Mino D, Maldonado-Julián H, Vidal-Cantú G et al. Efficacy and safety of 4-aminopyridine in patients with long-term spinal cord injury: a randomized, double-blind, placebo-controlled trial. Pharmacotherapy 2003; 23: 823-834.

42 Goodman AD, Brown TR, Krupp LB, Schapiro RT, Schwid SR, Cohen $\mathrm{R}$ et al. Sustained-release oral fampridine in multiple sclerosis: a randomised, double-blind, controlled trial. Lancet 2009; 373: 732-738.

43 Nógrádi A, Szabó A, Pintér S, Vrbová G. Delayed riluzole treatment is able to rescue injured rat spinal motoneurons. Neuroscience 2007; 144: 431-438.

44 Schwab J. Experimental strategies to promote spinal cord regeneration - an integrative perspective. The 7th International Symposium on Experimental Spinal Cord Repair and Regeneration. Brescia, Italy, 2009.

45 Byrnes KR, Waynant RW, Ilev IK, Wu X, Barna L, Smith K. et al. Light promotes regeneration and functional recovery and alters the immune response after spinal cord injury. Lasers Surg Med 2005; 36: 171-185.

46 von Wild K, Rabischong P, Brunelli G, Benichou M, Krishnan K. Computer added locomotion by implanted electrical stimulation in paraplegic patients (SUAW). Acta Neurochir Suppl 2002; 79: 99-104.

47 Shapiro S, Borgens R, Pascuzzi R, Roos K, Groff M, Purvines S et al. Oscillating field stimulation for complete spinal cord injury in humans: a phase 1 trial. J Neurosurg Spine 2005; 2: 3-10.

48 Belci M, Catley M, Husain M, Frankel HL, Davey NJ. Magnetic brain stimulation can improve clinical outcome in incomplete spinal cord injured patients. Spinal Cord 2004; 42: 417-419.

49 Lapointe NP, Guertin PA. Synergistic effects of D1/5 and 5-HT1A/ 7 receptor agonists on locomotor movement induction in complete spinal cord-transected mice. J Neurophysiol 2008; 100: 160-168.

50 Jordan L. Brainstem and spinal neural systems for the initiation of locomotion. Summer School for the Biological Treatment of Chronic Spinal Cord Injury; October 2008; Vienna, Austria, 2008.

51 Deliagina T. Spinal and supraspinal postural networks. Summer School for the Biological Treatment of Chronic Spinal Cord Injury. October 2008; Vienna, Austria, 2008.

52 Rossignol S. CPG in the recovery of locomotion after partial spinal cord lesions. Summer School for the Biological Treatment of Chronic Spinal Cord Injury; October 2008; Vienna, Austria, 2008.

53 Lyalka VF, Musienko PE, Orlovsky GN, Grillner S, Deliagina TG. Effect of intrathecal administration of serotoninergic and noradrenergic drugs on postural performance in rabbits with spinal cord lesions. J Neurophysiol 2008; 100: 723-732.

54 Dasen JS, Tice BC, Brenner-Morton S, Jessell TM. A Hox regulatory network establishes motor neuron pool identity and target-muscle connectivity. Cell 2005; 123: 477-491.

55 Song M-R, Pfaff SL. Hox genes: the instructors working at motor pools. Cell 2005; 123: 363-365.
56 Vaynman SS, Ying Z, Yin D, Gomez-Pinilla F. Exercise differentially regulates synaptic proteins associated to the function of BDNF. Brain Res 2006; 1070: 124-130.

57 Onose G, Padure L (eds). Compendiu de Neuroreabilitare - la adulti, copii si varstnici (Compendium of Neurorehabilitation-In Adults, Children and Elderly), Cap. 2. Editura Universitara Carol Davila: Bucharest, 2008, pp 21-87.

58 Onose G, Anghelescu A, Chendreanu C, Mihailescu C, Lapadat M, Mihaescu A-S et al. Standing and gait recovery in hemiplegic patients: outcome comparative analysis between postural reflexes/reactions stimulation versus classical prorioceptive facilitation method. The 31st National Annual Congress of Physical and Rehabilitation Medicine, Poiana Braşov, Romania, 29 October1 November 2008, 2009.

59 Hultborn H. Plasticity at motoneuronal level following spinal cord lesions. Summer School for the Biological Treatment of Chronic Spinal Cord Injury. October 2008; Vienna, Austria, 2008.

60 Dobkin B, Apple D, Barbeau H, Basso M, Behrman A, Deforge D et al. Weight-supported treadmill vs over-ground training for walking after acute incomplete SCI. Neurology 2006; 66: 484-493.

61 Lavrov I, Dy CJ, Fong AJ, Gerasimenko Y, Courtine G, Zhong H et al. Epidural stimulation induced modulation of spinal locomotor networks in adult spinal rats. J Neurosci 2008; 28: 6022-6029.

62 Minassian K, Jilge B, Rattay F, Pinter MM, Binder H, Gerstenbrand $\mathrm{F}$ et al. Stepping-like movements in humans with complete spinal cord injury induced by epidural stimulation of lumbar cord: electromyographic study of compound muscle action potentials. Spinal Cord 2004; 42: 401-416.

63 Minassian K, Persy I, Rattay F, Pinter MM, Kern H, Dimitrijevic MR. Human lumbar cord circuitries can be activated by extrinsic input tonic to generate locomotor-like activity. Hum Mov Sci 2007; 26: 275-295.

64 Brunelli G, von Wild KRH. Unsuspected plasticity of single neurons after connection of the cortico-spinal tract with peripheral nerves in spinal cord lesions. Korean J Neurosurg 2009; 46: 1 .

65 Von Wild KRH, Brunelli GA. Restoration of paraplegics with aid of autologous bypass grafts for direct neurotisation of muscles by upper motor neurons-the future: surgery of the spinal cord? Acta Neurochir Suppl 2003; 87: 107-112.

66 Pearse DD, Pereira FC, Marcillo AE, Bates ML, Berrocal YA, Filbin MT et al. cAMP and Schwann cells promote axonal growth and functional recovery after spinal cord injury. Nat Med 2004; 10: 610-616.

67 Fouad K, Schnell L, Bunge MB, Schwab ME, Liebscher T, Pearse DD. Combining Schwann cell bridges and olfactory-ensheathing glia grafts with chondroitinase promotes locomotor recovery after complete transection of the spinal cord. J Neurosci 2005; 25: 1169-1178.

68 Houle JD, Tom VJ, Mayes D, Wagoner G, Phillips N, Silver J. Combining an autologous nervous system bridge and matrix modification by chondroitinase allows robust, functional regeneration beyond a hemisection lesion of the adult rat spinal cord. J Neurosci 2006; 26: 7405-7415. 